Abstract G281(P) Table 1 Problems reported

\begin{tabular}{|c|c|c|}
\hline Interview question & Themes & Sub-themes \\
\hline \multirow[t]{9}{*}{ General problems produced by living in a refugee camp } & Education & Classrooms \\
\hline & & School life and location \\
\hline & Camp setting & Lack of electricity \\
\hline & & Lack of water \\
\hline & & Enclosed camp \\
\hline & & Financial problems \\
\hline & & Long waiting hours at the hospital \\
\hline & Daily routine & Boredom \\
\hline & & Social life in the camp \\
\hline \multirow[t]{8}{*}{ Most importnat mental health and psychosocial problems } & Child protection issues & Child marriage \\
\hline & & Child labour \\
\hline & Ongoing worries & Security \\
\hline & & Violence and bullying \\
\hline & & Domestic abuse \\
\hline & & Fears about the future \\
\hline & Feelings of sadness & Missing Syria \\
\hline & & Bereavement over people left behind \\
\hline
\end{tabular}

Conclusion Participants suffer a variety of psychosocial problems, mostly related to camp structure. However, this does not necessarily imply that they suffer from a mental disorder. With few validated assessment tools and great psychosocial distress, it is imperative to validate more tools to screen for mental disorders. Strengthening existing coping mechanisms such as social cohesion can help buffer many aspects of their distress. Finally, future research to substantiate this study's findings is required.

\section{G282(P) CAUSES OF MORTALITY IN A SIERRA LEONEAN DISTRICT HOSPITAL NEONATAL UNIT}

S Kirolos, J Sesay. Neonatal Unit, Kabala Government Hospital, Kabala, Koinadugu District, Sierra Leone

\subsection{6/archdischild-2018-rcpch.274}

Introduction Neonates are one of the most vulnerable groups of patients worldwide, and this is even more so in lowresource settings. In 2015, neonatal mortality in Sierra Leone was estimated at 35/1,000 live births. Although impossible to determine exactly, it is thought that $45 \%$ of under-five mortality worldwide occurs in the neonatal group. Basic neonatal care can make a significant difference in reducing morbidity and mortality.

Aim To ascertain the main causes of mortality in a Sierra Leonean district hospital neonatal unit.

Method Neonatal admissions over a four-month period from 1 st March 2017 to 30th June 2017 were retrospectively analysed. Data collected included demographics, main diagnosis, treatment and final outcome.

Results Over the study period there were 97 admissions to the neonatal unit. $20.6 \%$ had a birth weight less than $2000 \mathrm{~g}$. Overall mortality was $21.6 \%$. Given the lack of reliable diagnostic investigations, the main causes of death were ascertained based on history and clinical assessment. Of the neonates that did not survive, $47.6 \%$ had hypoxic ischaemic encephalopathy, defined as the need for resuscitation at birth and abnormal neurological examination thereafter. Prematiruty accounted for $23.8 \%$ of deaths, and this was determined by known gestation or clinical appearance of the neonate at birth when exact gestation was not known. The median birth weight of the premature neonates who died was $1005 \mathrm{~g}$, with more than half of them also having severe respiratory distress. Presumed sepsis accounted for 19\% of deaths.

Conclusion This is a report of the main causes of neonatal mortality in a neonatal unit in a Sierra Leonean district hospital. At the time of data collection, this unit was supported by an international non-government organisation and the average in-hospital birth rate was 90 live births per month. Almost half of deaths were secondary to hypoxic ischaemic encephalopathy, with the remainder being related to prematurity and its complications or presumed sepsis. Interventions aimed at reducing this burden of mortality include national neonatal resuscitation training, emphasis on prompt delivery of broad spectrum antibiotics for neonates with signs of early onset sepsis and basic care of the preterm infant, including kangaroo mother care.

\section{G283(P) IMPLEMENTATION OF EMERGENCY TRIAGE ASSESSMENT AND TREATMENT (ETAT+) IN A SIERRA LEONEAN DISTRICT HOSPITAL}

S Kirolos, I Sesay. Paediatric Department, Kabala Government Hospital, Kabala, Sierra Leone

\subsection{6/archdischild-2018-rcpch.275}

Introduction Paediatric under-five mortality in Sierra Leone is one of the highest worldwide at 114/1000 live births in 2016 . A history of civil war and viral haemorrhagic fever outbreak has impeded growth and development of the country's health system. With a lack of resources and very few paediatric trained staff, children's health care in Sierra Leone is greatly disadvantaged. The ETAT+protocols have been developed to improve emergency care of paediatric patients in low-resource settings.

Aim To ascertain the impact of implementing national ETAT + protocols on the quality of care and mortality in malaria admissions in a Sierra Leonean district hospital.

Methods Data on all paediatric malaria admissions were collected over a four-month period between 01/03/17 and 30/06/ 